\title{
Guide de présentation des rapports sur les commentaires
}

Correspondance : ccdr-rmtc@phac-aspc.gc.ca

Les commentaires sont des articles d'opinion destinés à stimuler la réflexion et le débat. lls déterminent un problème, le mettent dans un contexte plus large, puis offrent des explications pour instruire, motiver ou formuler une opinion. Habituellement, ils ont une longueur de 1000 à 1500 mots et comportent 10 à 15 références.

Les commentaires ont un titre engageant et un résumé du texte de 150 à 200 mots.

L'introduction compte habituellement deux à quatre paragraphes, qui déterminent un problème et expliquent pourquoi il est important. L'introduction exprime une position et décrit les principaux arguments pour appuyer cette position.

Le corps du commentaire compte cinq à huit paragraphes et fournit une analyse de la question. Un ou deux paragraphes peuvent être fournis pour développer chacun des arguments et tenir compte des points forts, des points faibles (ou contre-arguments), ainsi que des implications relatives aux politiques et aux pratiques. Les affirmations sont soutenues par des documents de référence clés. Des exemples peuvent être utilisés pour illustrer ou appuyer un point. Un tableau résumant les principaux renseignements peut être utile.

La conclusion compte un ou deux paragraphes. Elle met l'accent sur le message clé des commentaires et fournit des recommandations pour l'avenir ou les étapes suivantes.

Comme pour toutes les soumissions, vérifier les Renseignements à l'intention des auteurs (publiés en janvier chaque année avec le premier numéro de chaque nouveau volume) du Relevé des maladies transmissibles au Canada (RMTC) pour les aspects généraux des exigences relatives à la préparation et à la soumission des manuscrits. 